\section{Lumbrical muscle tear: clinical presentation, imaging findings and outcome}

\author{
Christoph Lutter ${ }^{1,2}$, Andreas Schweizer ${ }^{3}$, Volker Schöffl ${ }^{1,4,5}$, \\ Frank Römer ${ }^{6}$ and Thomas Bayer ${ }^{6}$
}

Journal of Hand Surgery

(European Volume)

2018, Vol. 43(7) 767-775

(C) The Author(s) 2018

Reprints and permissions:

sagepub.com/journalsPermissions.nav DOI: $10.1177 / 1753193418765716$ journals.sagepub.com/home/jhs

@SAGE

\begin{abstract}
The incidence of lumbrical muscle tear is increasing due to the popularity of climbing sport. We reviewed data from 60 consecutive patients with a positive lumbrical stress test, including clinical examination, ultrasound and clinical outcomes in all patients, and magnetic resonance imaging in 12 patients. Fifty-seven patients were climbers. Lumbrical muscle tears were graded according to the severity of clinical and imaging findings as Grade I-III injuries. Eighteen patients had Grade I injuries (microtrauma), 32 had Grade II injuries (muscle fibre disruption) and 10 had Grade III injuries (musculotendinous disruption). The treatment consisted of adapted functional therapy. All patients completely recovered and were able to return to climbing. The healing period in Grade III injuries was significantly longer than in the patients with Grade I or II injuries $(p<0.001)$. We recommend evaluation of specific clinical and imaging findings to grade the injuries and to determine suitable therapy.
\end{abstract}

Level of evidence: IV

Keywords

Climbing, lumbrical muscle tear, Quadriga, magnetic resonance imaging, ultrasound

Date received: 21st November 2017; revised: 20th February 2018; accepted: 27th February 2018

\section{Introduction}

Lumbrical muscle tears are rare injuries of the palm caused by a so-called 'quadriga effect', which describes a shear injury resulting from pathologic stress to the two origins of the bipennate lumbrical muscle (Schweizer, 2003; Verdan and Poulenas, 1975). This pathomechanism results from gripping positions of the hand in which one or two fingers are extended while the neighbouring fingers are actively flexed (Schweizer, 2003; Verdan and Poulenas, 1975). Lumbrical muscles are known to have a wide range of anatomical variation (Palti and Vigler, 2012; Parminder, 2013). Injuries typically occur within the third or fourth lumbrical muscles, since these are the only muscles that have a bipennate origin in almost all individuals (Palti and Vigler, 2012; Parminder, 2013; Wang et al., 2016).

The injury predominantly affects rock-climbing athletes, as they frequently use the aforementioned gripping position of the hand (Schöffl and Schöffl, 2007; Schöffl et al., 2012; Schöffl et al., 2015;
Schweizer, 2003; Wang et al., 2016). The rising popularity of this sport has led to an increased incidence of lumbrical tears in recent years (Lapegue et al., 2015; Wang et al., 2016). However, since the publication of Schweizer (2003), only a few case reports that describe the pathomechanism and mention the

\footnotetext{
${ }^{1}$ Department of Sports Orthopaedics, Sports Medicine, Sports Traumatology, Klinikum Bamberg, Germany

${ }^{2}$ CVPath Institute, Gaithersburg, USA

${ }^{3}$ Hand Surgery Department, University of Zurich, Zurich, Switzerland

${ }^{4}$ Department of Trauma and Orthopaedic Surgery, Friedrich Alexander University, Erlangen-Nuremberg, Germany

${ }^{5}$ Department of Emergency Medicine, University of Colorado School of Medicine, Denver, CO, USA

${ }^{6}$ Department of Radiology, Friedrich Alexander University Erlangen-Nuremberg, Germany

Corresponding Author:

Christoph Lutter, Department of Sports Orthopaedics, Sports Medicine, Sports Traumatology, Department of Orthopaedics and Traumatology, Klinikum Bamberg, Germany.

Email: christoph.lutteragooglemail.com
} 
anatomical considerations have been published (Lapegue et al., 2015; Murphy and Chernofsky, 1999; Wang et al., 2016). Therefore, recommendations for diagnostic and therapeutic approaches in the treatment of affected patients are lacking. In addition, there is a paucity of data regarding the outcomes and prognosis of these injuries.

We serve as a referral centre for rock-climbing specific injuries and therefore receive a high number of patients suffering from acute lumbrical muscle tears. In order to improve and standardize the treatment, all cases since 2009 have been treated according to clearly defined diagnostic and therapeutic algorithms. The purpose of this study was to retrospectively analyse data from 60 consecutive patients with lumbrical muscle tears with respect to clinical presentation, imaging findings, therapy and outcomes.

\section{Materials and methods}

\section{Patients}

Between 2009 and 2017, we investigated 60 consecutive patients suffering from pain in the palm provoked by a shift of the deep flexor tendons of the adjacent fingers (Table 1), which reflects the 'quadriga effect' (Figure 1). Only patients with a positive clinical stress test, as described by Schweizer (2003), were included in this study (Figure 2). Patients were seen and treated in our orthopaedic/trauma surgery centre, which works as a referral centre for climbing related injuries. Patients reporting persistent pain for more than 3 months were classified as 'chronic before therapy', whereas all other patients were classified as 'acute before therapy'. The study was approved by the institutional review board and all patients provided written informed consent.

\section{Diagnostic algorithm}

A grading system was developed based on the assessment of patients not included in the current study sample, according to the severity of clinical and imaging findings (Figure 3).

Grade I. Micro trauma in the lumbrical muscle. Positive clinical stress test with no pathological finding on ultrasound (US) (i.e. no detectable oedema or bleeding in the lumbrical muscles, no tenosynovitis).

Grade II. Lumbrical muscle fibre disruption without tendon involvement. US showing muscle oedema in the lumbrical muscle with/without adjacent tenosynovitis of the flexor tendons.

Grade III. Musculotendinous disruption in the lumbrical muscle. Magnetic resonance imaging (MRI) evidence of tendon discontinuity at the flexor digitorum profundus or flexor digitorum superficialis tendon origin. Grade III was diagnosed whenever a lumbrical muscle tendon discontinuity was seen, regardless of the extent of muscle oedema, haematoma or adjacent tenosynovitis of the flexor tendons.

\section{Imaging}

US examination was performed on all patients using a GE logic E9 ${ }^{\circledR}$ (General Electrics Healthcare ${ }^{\circledR}$, Buckinghamshire, UKJ with a linear $18 \mathrm{MHz}$ probe on the palm.

Table 1. Patient demographics, severity of injuries and recovery after injury.

\begin{tabular}{|c|c|c|c|c|}
\hline Demographics and recovery & Overall & Grade I & Grade II & Grade III \\
\hline Number of patients & $60^{a}$ & $18(30 \%)$ & $32(53 \%)$ & $10^{\mathrm{a}}(17 \%)$ \\
\hline Age (years) & $\begin{array}{l}30.3(7.7) \\
\text { (range 18.0-46.5) }\end{array}$ & $\begin{array}{l}30.3(7.7) \\
\text { (range 19.0-46) }\end{array}$ & $\begin{array}{l}29.5(7.6) \\
\text { (range 18-45) }\end{array}$ & $\begin{array}{l}32.7 \text { (8.7) } \\
\text { (range 23-45) }\end{array}$ \\
\hline Sex (men:women) & $45: 15$ & $14: 4$ & $26: 6$ & $5: 5$ \\
\hline Acute/chronic presentation & $57 / 3$ & $18 / 0$ & $29 / 3$ & $10 / 0$ \\
\hline Affected side (R:L) & $32: 28$ & $8: 10$ & $21: 11$ & $3: 7$ \\
\hline Handedness (R:L) & $53: 7$ & $16: 2$ & $28: 4$ & $9: 1$ \\
\hline Affected finger (middle:ring) & $16: 44$ & $5: 13$ & $9: 23$ & 2: 8 \\
\hline Healing period (weeks) & $\begin{array}{l}7 \text { (2) } \\
\text { (range 3-12) }\end{array}$ & $\begin{array}{l}5(1) \\
\text { (range 4-8) }\end{array}$ & $\begin{array}{l}6(2) \\
\text { (range 3-9) }\end{array}$ & $\begin{array}{l}10(2) \\
\text { (range 8-12) }\end{array}$ \\
\hline UIAA ${ }^{b}$ Climbing level (among climbers) & $8.8(1.1)$ & $8.4(0.7)$ & $8.9(1.2)$ & $9.7(0.8)$ \\
\hline
\end{tabular}

${ }^{a}$ Three patients in these categories are non-climbers.

${ }^{b}$ Scale ranging from 2 to 11 (Union Internationale des Associations d'Alpinisme (UIAA), www.theuiaa.org). 


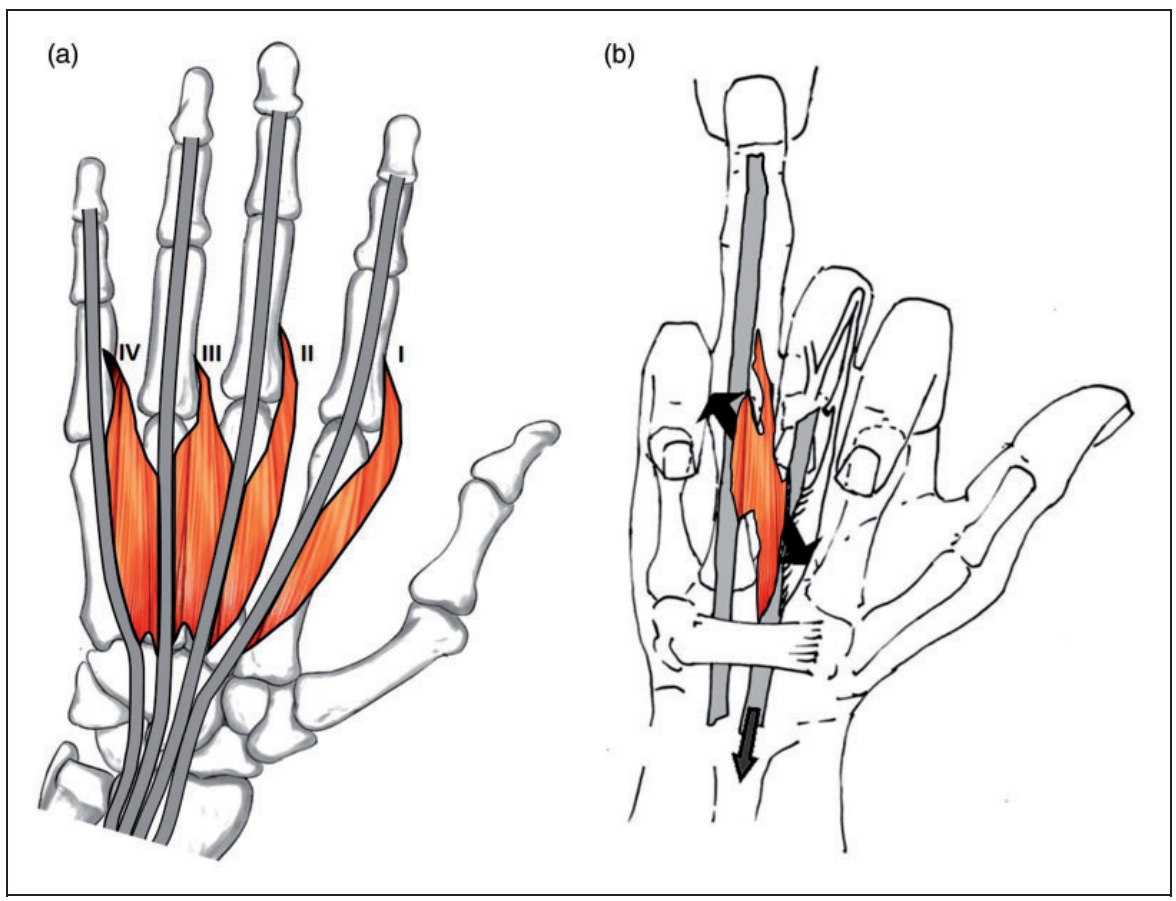

Figure 1. (a) Normal anatomy of the four lumbrical muscles (I/II unipennate, III/IV bipennate) and (b) pathomechanism of lumbrical muscle tears referred to as 'quadriga effect'.

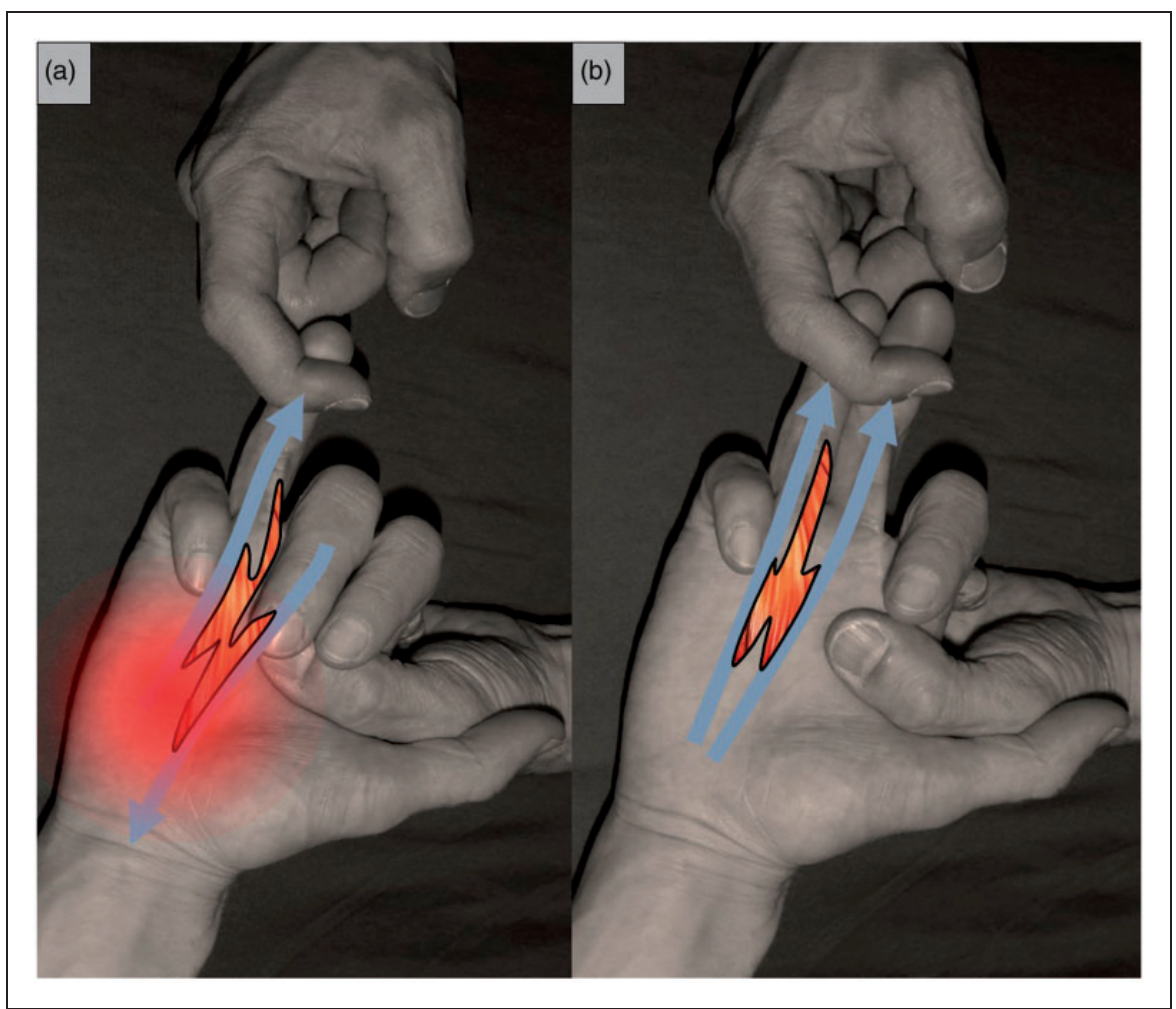

Figure 2. Stress test of lumbrical muscle tear. (a) Positive stress test: provocation of pain with passive extension of the ring finger while patient aims to close his fist. (b) No symptoms can be provoked when the middle finger is extended simultaneously, as the quadriga effect of the third lumbrical muscle disappears due to non-separation of the bipennate origins. 


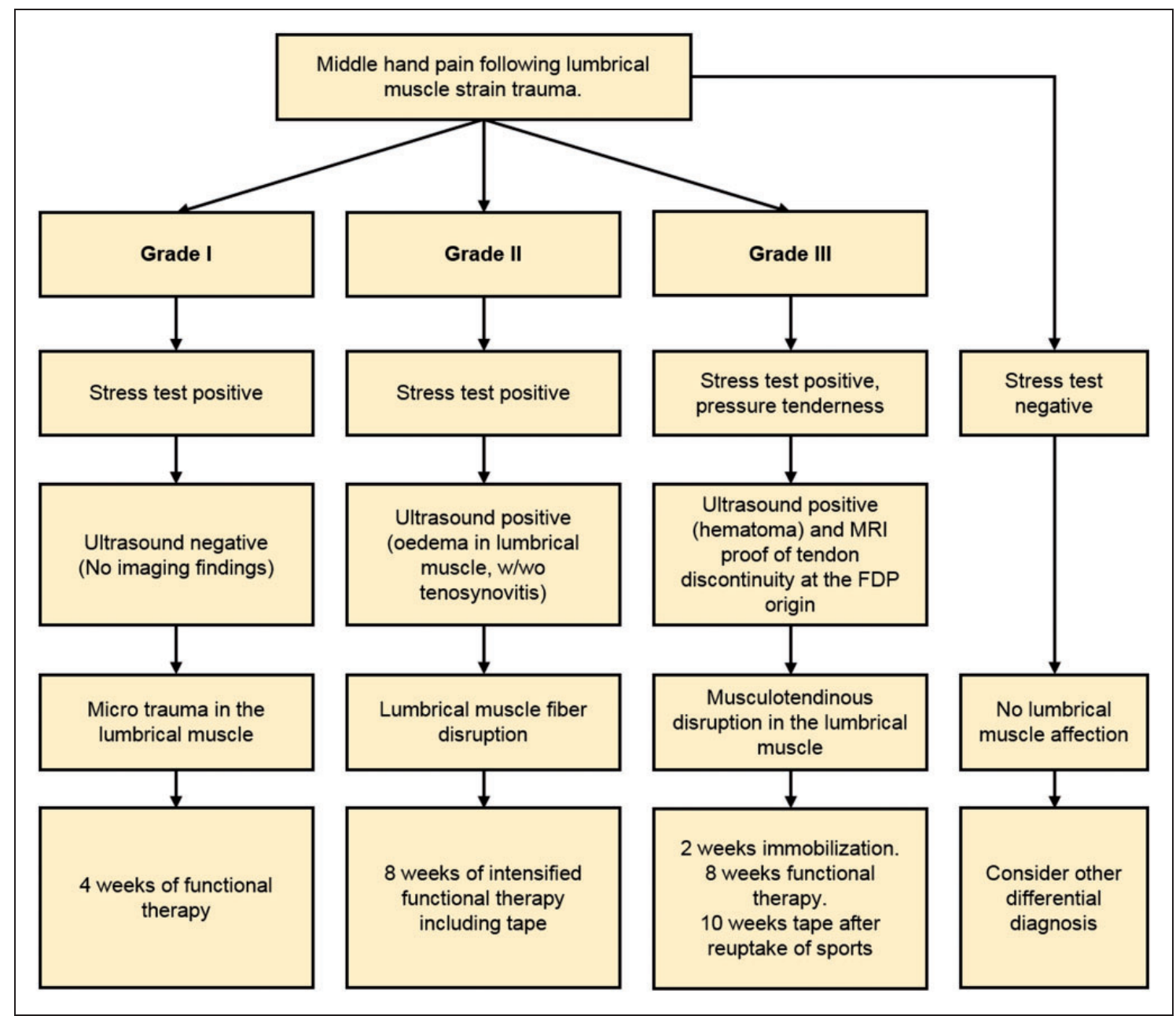

Figure 3. Algorithm of diagnostic and therapeutic treatment for lumbrical muscle injuries based on the severity of clinical and imaging findings.

Magnetic resonance imaging (MRI) was performed for further evaluation in $n=12 / 60$ cases because clinical examination showed distinct point tenderness of the palm with a positive stress test result and US examination showed definite lumbrical muscle haematoma (Figure 4 and Supplementary material Figure S1). A 3 Tesla whole body scanner (Magnetom Skyra, Siemens Healtheneers, Erlangen, Germanyl was used.

The imaging was analysed by an experienced musculoskeletal radiologist (T.B.) on a commercially available picture archiving and communication system monitor (PACS SyngoPlaza, Siemens Healthineers, Erlangen, Germanyl. For Grade III injuries, the distance of lumbrical muscle separation was determined by measurements between the flexor digitorum profundus and the lumbrical muscle tendon laccuracy of measurements $0.1 \mathrm{~mm}$ ).

\section{Therapeutic algorithm}

All patients were treated conservatively according to the algorithm shown (Figure 3).

Grade I. Patients were treated with functional therapy (free range of motion, avoidance of load on isolated fingers) for 4 weeks. Carefully dosed and regular stretching of the lumbrical muscle was advised in order to prevent the development of excessive scar tissue (Chicarilli et al., 1986; Tan et al., 2002). Stretching consisted of a careful passive movement into the so-called intrinsic minus position (Liss, 2012) (Supplementary material 2(a)). Once this stretching exercise could be performed without pain, further stretching was started in the affected lumbrical muscle using a carefully dosed and pain-free stimulation of the quadriga effect with passive extension of the affected finger, while 

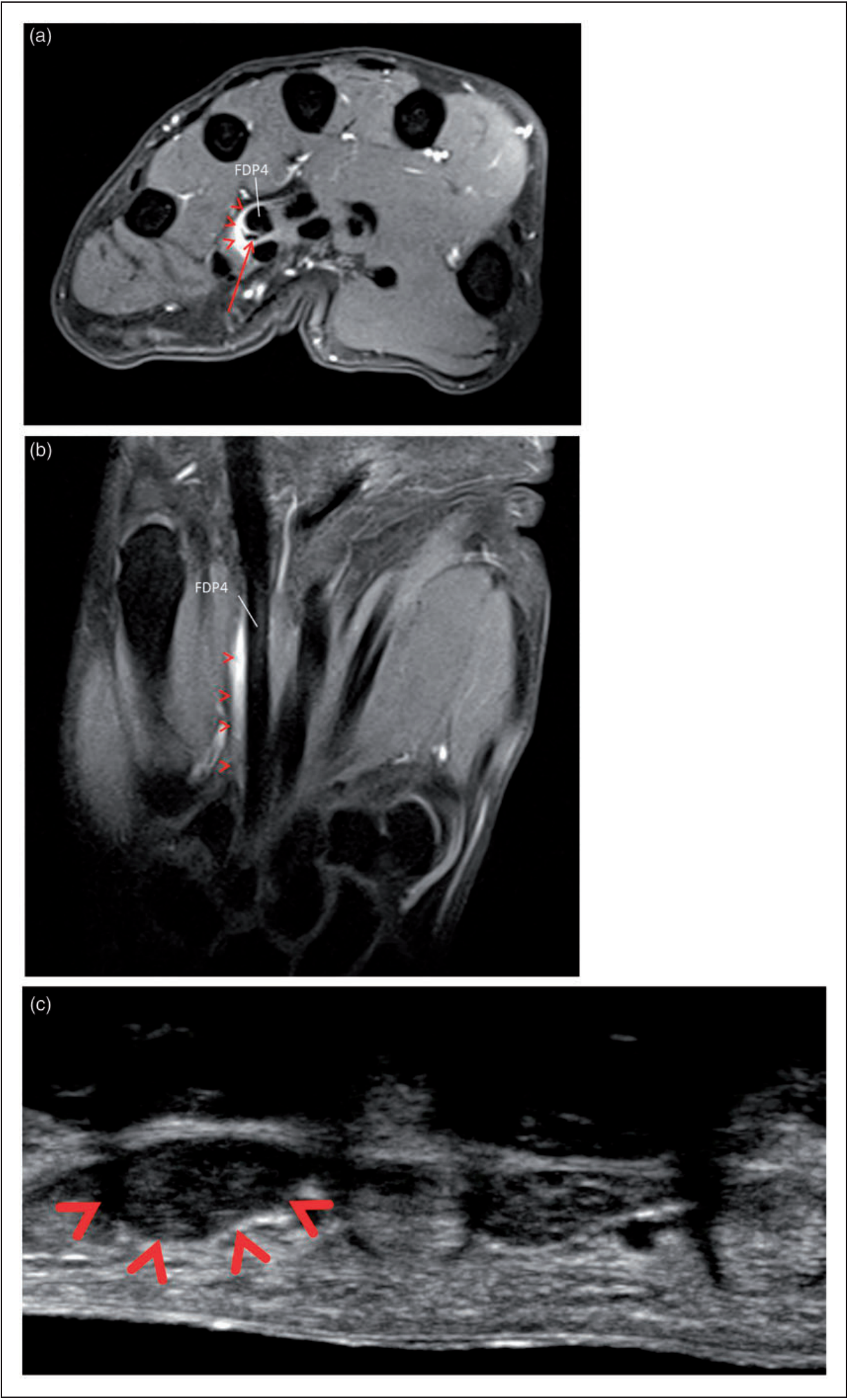

Figure 4. A 42-year-old female patient suffering from acute Grade III lumbrical muscle injury of the left hand. T1 weighted fat saturated MRI in the axial (a) and coronal (b) directions after intravenous contrast enhancement showing separation of the fourth lumbrical muscle tendon (red arrow (a)) from the musculotendinous flexor digitorum profundus origin of the ring finger only on the ulnar side. Note the surrounding inflammatory reaction in the lumbrical muscle (red arrowheads, (a) and (b)), representing only a half Theta sign. (c) US also shows swelling and hyper-echogenic reflections representing haematoma in the fourth lumbrical muscle (red arrowheads, (c)). 

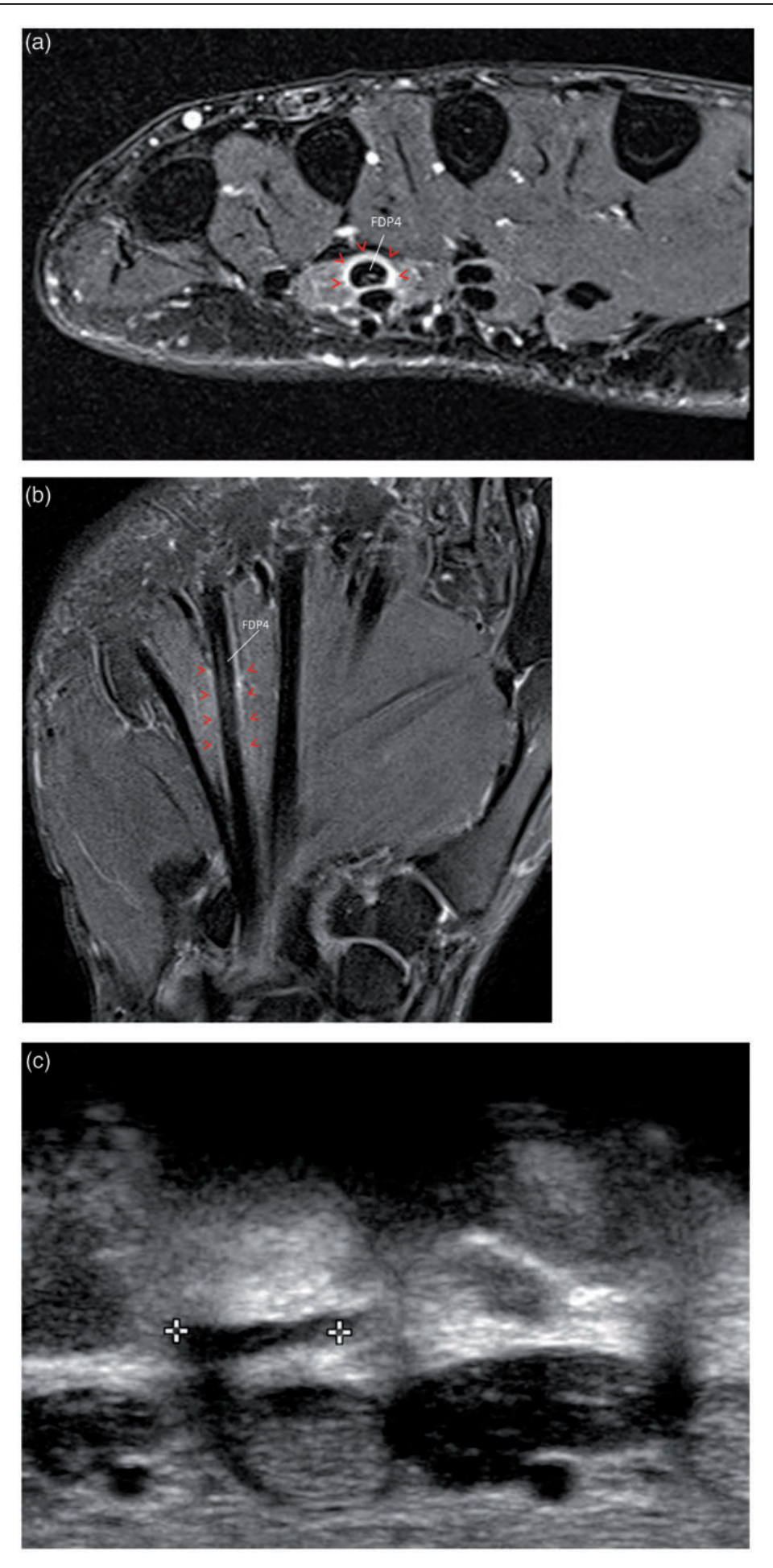

Figure 5. A 44-year-old male patient suffering from acute Grade II lumbrical muscle injury of the left hand. Proton density weighted fat saturated MRI in the axial (a) and coronal (b) directions showing inflammatory reaction in the third and fourth lumbrical muscle (red arrowheads, (a) and (b) on both sides of the flexor digitorum profundus (full Theta sign). US (c) shows fluid (white crosses) along the 4th flexor digitorum superficialis and flexor digitorum profundus tendon. MRI was performed in this case because of an atypical intensity of symptoms with pressure tenderness along the 4th finger flexor tendons in order to exclude a lumbrical muscle tendon separation. 
the neighbouring fingers were actively flexed (Supplementary material Figure S2(b)). If soreness was still present at the first follow-up investigation (usually after 6 weeks), functional therapy was resumed for another 4 weeks. Immobilization was not recommended.

Grade II. Patients were treated with an intensified functional therapy. The so called 'buddy taping' technique (Vadstrup et al., 2014; Won et al., 2014), using loosely applied circular tape around the proximal and the middle phalanx of the affected fingers, was allowed for a maximum of 8 weeks if required for pain reduction during routine daily activities. Otherwise, a free range of motion was allowed, but loading of isolated fingers was to be avoided. The patients were advised to start careful stretching similar to Grade I injuries, as soon as symptoms had reduced and the exercises could be performed with no pain.

Grade III. Patients received temporary splintimmobilization (distal forearm to the proximal interphalangeal joints of the middle, ring and little fingers) for 2 weeks, followed by an intensified functional therapy as for Grade II injuries for another 8 weeks. In this group, taping was advised during climbing for at least 10 weeks after recommencing sports.

\section{Autologous conditioned plasma infiltration}

In three lumbrical muscle injuries that occurred in professional athletes competing on a World Cup level, additional local infiltration therapy was performed in order to shorten the recovery time for a faster 'return to competition'. In those cases, we repetitively injected Autologous Conditioned Plasma ${ }^{\circledR}$ (ACP, Arthrex, Naples, FL, USAl in the affected region; this approach was based on positive experience with this technique in a previous study (Schöffl et al., 2017). In one additional patient of an average climbing performance level, who was suffering from a chronic lumbrical muscle injury, local infiltration was performed because of lack of progress with functional therapy.

\section{Outcome}

All patients were seen for follow-up investigations 6 and 12 weeks after the initial consultation. Complete healing was defined as when patients were fully free of symptoms at follow-up. The length of the healing period was recorded. For rock climbing athletes this meant the time when the pre-injury climbing level was regained, and for non-climbers the time, when the individuals were free of symptoms during everyday activities.

\section{Statistical analysis}

Unless stated otherwise, data were expressed as mean (standard deviation). $P$-values $<0.05$ were considered statistically significant. A $t$-test or Rank-sum test was used to determine differences between the three injury severity grades.

\section{Results \\ Incidence of quadriga effect}

All 60 patients reported onset of pain after excessive loading of isolated ring or middle fingers according to the quadriga effect described by Schweizer (2003), without evidence of other causes, such as inflammatory factors or tumour (Table 1). Clinical examination revealed pain in the palm or point tenderness over the respective flexor tendons and/or lumbrical muscles. Apart from these findings, clinical examination was unremarkable.

\section{Grading and locations}

Three patients diagnosed with a Grade III injury were non-climbers (Table 1). Among the climbing athletes (57 patients), the mean rock-climbing experience was 11 years (7) (range 2-30). Three patients had a chronic condition at time of first presentation with symptoms having been present for 4 to 16 months. All three were diagnosed with a Grade II injury and were free of symptoms at the second follow-up 112 weeks).

\section{Locations of injuries in US images and MRI}

US located the injuries in the fourth lumbrical muscle in 23 of the 32 Grade II cases and in the third lumbrical muscle in nine (Figures 4(c) and 5(c), Supplementary Figure S1(c)). MRI located musculotendinous disruption in the fourth lumbrical muscle in eight of the 10 of the Grade III cases and in the third lumbrical muscle in two (Figures 4 (a) and (b) and 5 (a) and (b), Supplementary Figure S1 (a) and (b)). The mean length of lumbrical muscle discontinuity was $1.1 \mathrm{~mm} \mathrm{(0.2).}$

\section{Outcomes}

The recovery and outcomes of patients in injury subgroups are given in Table 1. All patients were free of pain after 12 weeks of conservative treatment. Statistical analysis revealed no significant differences between the three sub-groups, except that the healing period for the Grade III injuries was 
significantly longer than in the two other sub-groups $(P<0.001)$.

\section{Discussion}

Our study confirms that symptoms from lumbrical muscle tears resolve after conservative therapy. Recovery takes longer in more severe injuries where there is musculotendinous discontinuity. The suggested pathomechanism of motion of the fingers, referred to as 'quadriga effect' (Schweizer, 2003; Verdan and Poulenas, 1975), was noted in all cases. There was no association of injury severity with patient age, gender or hand dominance. However, more severe injuries occurred in patients who climbed on a higher performance level.

The diagnostic and therapeutic algorithms of our study were implemented in order to improve the differentiation of injury severity and the standardization in the treatment of patients with acute lumbrical tears. Our injury classification is based on a combination of radiological and clinical findings, which were the basis for subsequent therapy decision-making. We believe that the early commencement of stretching exercises was beneficial in preventing the development of interosseous-lumbrical adhesions (Chicarilli et al., 1986; Tan et al., 2002). It is noteworthy that conservative therapy was successful even in the Grade III sub-group, in which MRI showed a musculotendinous disruption. This is most likely explained by the limited distances of tendon separation in the lumbrical origin and the fact that no wavelike retracted tendon configurations were present.

Musculotendinous disruption as found in the Grade III tears is an imaging finding, which has not been described in previous studies. We consider this finding as therapeutically relevant with temporary immobilization being required. This policy was supported by the longer time to full recovery for these injuries compared with Grade II and I injuries. Our study confirmed the so-called 'theta sign', as described by Wang et al. (2016), which refers to a reactive tenosynovitis resembling the Greek letter $\theta$ (theta), surrounding the flexor tendons of the affected finger in $50 \%$ of the patients. Beyond that, our imaging revealed a great variety of location and extent of muscle oedema, tenosynovitis and/or localization of the lumbrical muscle tear.

Our experience with chronic lumbrical tears is limited and we recommend diagnosing and treating the chronic cases depending on the individual presentation. Our data suggests that a lumbrical muscle tear may become a chronic clinical problem if left untreated, which implies that there is a necessity for a timely diagnosis and early treatment.

Lumbrical muscle tears in none climbers are rare. We suggest that the diagnostic and therapeutic procedures in these patients should be identical to those described above for climbers. However, the risk of misdiagnosis may be increased for the non-climbing sub-group, especially when no definite trauma is recalled and when the initial clinical examination is not performed by a trauma specialist familiar with the injury.

We found that patients with lumbrical muscle tears have a typical clinical presentation and that due to our inclusion criteria (positive stress test), imaging did not reveal any other potential differential diagnoses, such as interosseous adhesions (Chicarilli et al., 1986; Tan et al., 2002), middle hand impingements, tenosynovitis of the flexor tendons, blunt flexor tendon disruptions (Drape et al., 1998; Lapegue et al., 2015; Schöffl et al., 2012), flexor tendon pulley ruptures (Schöffl and Schöffl, 2007) or tumours.

One major limitation of this study is that MRI was only available in a minority of our patients and as such, the differences in diagnostic performance for detection of lumbrical muscle injuries could not be studied. In our study, structural Grade III injuries could have possibly been missed in patients with low-grade clinical injuries. MRI may be more sensitive, but we suggest performing an US examination as the first imaging procedure due to the greater availability and cost reduction. We feel that this approach might also be appropriate for other institutions. Another limitation of this study is that we did not systematically examine the performance of diverging or enhanced treatment strategies beyond our standard approach for the three diagnostic patient sub-groups. We recommend performing a further study with a prospective randomized design for clarifying these open questions.

In summary, the overall outcome in our series of lumbrical muscle tears was positive and uncomplicated using a conservative treatment strategy according to our algorithms. We recommend evaluation of specific clinical and imaging findings to grade the severity of these injuries and to determine the suitable course of therapy.

Acknowledgements The authors thank Rosamunde Pare for English language support.

Contributors CL, AS, VS, FR and TB designed the study, monitored the data collection, analysed and interpreted the data and drafted the article. CL, AS and VS were responsible for clinical treatment of the patients including examinations. TB, VS and AS interpreted the MRIs and USs. 
Declaration of conflicting interests The authors declared no potential conflicts of interest with respect to the research, authorship, and/or publication of this article.

Ethical approval The study was approved by the institutional review board and all patients provided written informed consent.

Funding The authors received no financial support for the research, authorship, and/or publication of this article.

Supplementary material Supplementary material is available at journals.sagepub.com/doi/suppl/10.1177/ 1753193418765716.

\section{References}

Chicarilli ZN, Watson HK, Linberg R, Sasaki G. Saddle deformity. Posttraumatic interosseous-lumbrical adhesions: review of eighty-seven cases. J Hand Surg Am. 1986, 11: 210-8.

Drape JL, Tardif-Chastenet de Gery S, Silbermann-Hoffman 0 et al. Closed ruptures of the flexor digitorum tendons: MRI evaluation. Skeletal Radiol. 1998, 27: 617-24.

Lapegue F, Andre A, Brun C et al. Traumatic flexor tendon injuries. Diagn Interv Imaging. 2015, 96: 1279-92.

Liss FE. The interosseous muscles: the foundation of hand function. Hand Clin. 2012, 28: 9-12.

Murphy RX Jr, Chernofsky MA. Tear of an anomalous lumbrical muscle as a source of pain in the hand. J Hand Surg Am. 1999, 24: 1078-82.

Palti R, Vigler M. Anatomy and function of lumbrical muscles. Hand Clin. 2012, 28: 13-7.
Parminder K. Morphological study of lumbricals - a cadaveric study. J Clin Diagn Res. 2013, 7: 1558-60.

Schöffl V, Heid A, Kupper T. Tendon injuries of the hand. World J Orthop. 2012, 3: 62-9.

Schöffl V, Popp D, Küpper T, Schöffl I. Injury trends in rock climbers: evaluation of a case series of 911 injuries between 2009 and 2012. Wilderness Environ Med. 2015, 26: 62-7.

Schöffl V, Willauschus W, Sauer F et al. Autologous conditioned plasma versus placebo injection therapy in lateral epicondylitis of the elbow: a double blind, randomized study. Sportverletz Sportschaden. 2017, 31: 31-6.

Schöffl VR, Schöffl I. Finger pain in rock climbers: reaching the right differential diagnosis and therapy. J Sports Med Phys Fitness. 2007, 47: 70-8.

Schweizer A. Lumbrical tears in rock climbers. J Hand Surg Br. 2003, 28: 187-9.

Tan V, Rothenfluh DA, Beredjiklian PK, Potter HG, Weiland AJ. Interosseous-lumbrical adhesions of the hand: contribution of magnetic resonance imaging to diagnosis and treatment planning. J Hand Surg Am. 2002, 27: 639-43.

Vadstrup LS, Jorring S, Bernt P, Boeckstyns ME. Base fractures of the fifth proximal phalanx can be treated conservatively with buddy taping and immediate mobilisation. Dan Med J. 2014, 61: A4882.

Verdan C, Poulenas I. Anatomic and functional relations between the tendons of the long palmar muscle and the long flexor muscle of the thumb at their crossing in the carpus. Ann Chir Plast. 1975, 20: 191-6.

Wang EH, Loftus WK, Bird SJ, Sampson MJ. Ring finger lumbrical origin strain: a case series with imaging findings. Skeletal Radiol. 2016, 45: 1729-34.

Won SH, Lee S, Chung CY et al. Buddy taping: Is it a safe method for treatment of finger and toe injuries? Clin Orthop Surg. 2014 6: $26-31$. 